International Journal of Pure and Applied Mathematics

Volume 104 No. 2 2015, 159-169

ISSN: 1311-8080 (printed version); ISSN: 1314-3395 (on-line version)

url: http://www.ijpam.eu

doi: http://dx.doi.org/10.12732/ijpam.v104i2.1

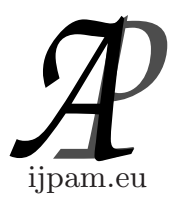

\title{
HYBRID ONE STEP BLOCK METHOD FOR THE SOLUTION OF FOURTH ORDER INITIAL VALUE PROBLEMS OF ORDINARY DIFFERENTIAL EQUATIONS
}

\author{
A.O. Adesanya ${ }^{1 \S}$, A.U. Fotta ${ }^{2}$, B. Abdulkadri ${ }^{3}$ \\ ${ }^{1}$ Department of Mathematics \\ Modibbo Adama University of Technology \\ Yola, Adamawa State, NIGERIA \\ ${ }^{2}$ Department of Mathematics \\ Adamawa State Polytechnic \\ Yola, Adamawa State, NIGERIA \\ ${ }^{3}$ Department of Mathematics \\ Federal College of Education \\ Yola, Adamawa State, NIGERIA
}

\begin{abstract}
We consider collocation and interpolation of the approximate solution at some selected grid and off grid points to give a system of nonlinear equations, solving for the unknown constants using Guassian elimination method and substituting into the approximate solution gives the continuous block method. We investigate the basic properties of the derived method, numerical examples show that the method is suitable for solving fourth order initial value problem of ordinary differential equations.
\end{abstract}

Key Words: collocation, interpolation, continuous block method, fourth order initial value problem, approximate solution

Received: August 5, 2013

(C) 2015 Academic Publications, Ltd. url: www.acadpubl.eu

${ }^{\S}$ Correspondence author 


\section{Introduction}

This paper considers approximate solution to problems in the form

$$
y^{i v}=f\left(x, y, y^{\prime}, y^{\prime \prime}, y^{\prime \prime \prime}\right)
$$

subject to

$$
y^{(k)}\left(x_{n}\right)=y_{n}^{(k)}, \quad k=0,1,2,3,
$$

where $x_{n}$ is the initial point, $y_{n}^{(k)}$ are the solutions at the initial point, $f$ is continuous within the interval of integration and satisfies the existence and uniqueness theorem given in [1].

Reduction of (1) to systems of first order ordinary differential equations has been established in literature not to be effective in terms of cost of evaluation, time of execution and approximation $[2,3,4,5]$.

Different basis function for the solution of higher order ordinary differential equations have been proposed in literature ranging from power series, Langrange polynomial, Newton's polynomial, Chebychev polynomial, backward differentiation formula, Fourier series to mention few. The choice of approximate solution depends on the nature of problem to be solved $[6,7,8,9]$.

Block method has equally been established in literature to be more effective than the predictor-corrector method in terms of accuracy, time of implementetion and cost of execution $[10,11]$.

Adopted one step approach which leads to hybrid method circumvents the Dahlquist stability barrier, gives better stability properties and approximation than the multistep method $[12,13]$.

In this paper, we combine the properties of hybrid and block method to develop one step block integrator for the solution of (1).

\section{Methods}

We consider approximate solution

$$
y(x)=\sum_{j=0}^{r+s-1} a_{j} x^{j},
$$

where $r$ and $s$ are the number of interpolation and collocation points respectively, $x \in[a, b], x^{j}$ are the polynomial basis function of degree $r+s-1 . a_{j}^{\prime} s \in \mathbb{R}$ are constants to be determined. 
Substituting the fourth derivative of (3) into (1) gives

$$
f\left(x, y, y^{\prime}, y^{\prime \prime}, y^{\prime \prime \prime}\right)=\sum_{j=0}^{r+s-1} j(j-1)(j-2)(j-3) a_{j} x^{j-4}
$$

Collocating (4) at $x_{n+s}, s=0\left(\frac{1}{6}\right) 1$ and interpolating (3) at $x_{n+r}, r=0\left(\frac{1}{6}\right) \frac{1}{2}$ gives a system of non linear equation of the form

$$
A X=U
$$

where

$$
\begin{gathered}
A=\left[\begin{array}{llllllllll}
a_{0} & a_{1} & a_{2} & a_{3} & a_{4} & a_{5} & a_{6} & a_{7} & a_{8}
\end{array}\right]^{T}, \\
U=\left[\begin{array}{ccccccccc}
y_{n} & y_{n+\frac{1}{4}} & y_{n+\frac{1}{2}} & y_{n+\frac{3}{4}} & f_{n} & f_{n+\frac{1}{4}} & f_{n+\frac{1}{2}} & f_{n+\frac{3}{4}} & f_{n+1}
\end{array}\right]^{T}, \\
X=\left[\begin{array}{ccccccccc}
1 & x_{\mathrm{n}} & x_{\mathrm{n}}^{2} & x_{\mathrm{n}}^{3} & x_{\mathrm{n}}^{4} & x_{\mathrm{n}}^{5} & x_{\mathrm{n}}^{6} & x_{\mathrm{n}}^{7} & x_{\mathrm{n}}^{8} \\
1 & x_{\mathrm{n}+\frac{1}{4}} & x_{\mathrm{n}+\frac{1}{4}}^{2} & x_{\mathrm{n}+\frac{1}{4}}^{3} & x_{\mathrm{n}+\frac{1}{4}}^{4} & x_{\mathrm{n}+\frac{1}{4}}^{5} & x_{\mathrm{n}+\frac{1}{4}}^{6} & x_{\mathrm{n}+\frac{1}{4}}^{7} & x_{\mathrm{n}+\frac{1}{4}}^{8} \\
1 & x_{\mathrm{n}+\frac{1}{2}} & x_{\mathrm{n}+\frac{1}{2}}^{2} & x_{\mathrm{n}+\frac{1}{2}}^{3} & x_{\mathrm{n}+\frac{1}{2}}^{4} & x_{\mathrm{n}+\frac{1}{2}}^{5} & x_{\mathrm{n}+\frac{1}{2}}^{6} & x_{\mathrm{n}+\frac{1}{2}}^{7} & x_{\mathrm{n}+\frac{1}{2}}^{8} \\
1 & x_{\mathrm{n}+\frac{3}{4}} & x_{\mathrm{n}+\frac{3}{4}}^{2} & x_{\mathrm{n}+\frac{3}{4}}^{3} & x_{\mathrm{n}+\frac{3}{4}}^{4} & x_{\mathrm{n}+\frac{3}{4}}^{5} & x_{\mathrm{n}+\frac{3}{4}}^{6} & x_{\mathrm{n}+\frac{3}{4}}^{7} & x_{\mathrm{n}+\frac{3}{4}}^{8} \\
0 & 0 & 0 & 0 & 24 & 120 x_{\mathrm{n}} & 360 x_{\mathrm{n}}^{2} & 840 x_{\mathrm{n}}^{3} & 1680 x_{\mathrm{n}}^{4} \\
0 & 0 & 0 & 0 & 24 & 120 x_{\mathrm{n}+\frac{1}{4}} & 360 x_{\mathrm{n}+\frac{1}{4}} & 840 x_{\mathrm{n}+\frac{1}{4}}^{3} & 1680 x_{\mathrm{n}+\frac{1}{4}}^{4} \\
0 & 0 & 0 & 0 & 24 & 120 x_{\mathrm{n}+\frac{1}{2}} & 360 x_{\mathrm{n}+\frac{1}{2}}^{2} & 840 x_{\mathrm{n}+\frac{1}{2}}^{3} & 1680 x_{\mathrm{n}+\frac{1}{2}}^{4} \\
0 & 0 & 0 & 0 & 24 & 120 x_{\mathrm{n}+\frac{3}{4}} & 360 x_{\mathrm{n}+\frac{3}{4}}^{2} & 840 x_{\mathrm{n}+\frac{3}{4}}^{3} & 1680 x_{\mathrm{n}+\frac{3}{4}}^{4} \\
0 & 0 & 0 & 0 & 24 & 120 x_{\mathrm{n}+1} & 360 x_{\mathrm{n}+1}^{2} & 840 x_{\mathrm{n}+1}^{3} & 1680 x_{\mathrm{n}+1}^{4}
\end{array}\right] .
\end{gathered}
$$

Solving (5) for the unknown constants, substituting into (3) gives continuous linear method

$$
\begin{array}{r}
y(t)=\sum_{j=o}^{1} \alpha_{j}(t) y_{n+j}+\alpha_{v}(t) y_{n+v}+h^{4}\left[\sum_{j=0}^{1} \beta_{j}(t) f_{n+j}+\left.\beta_{v}(t) f_{n+v}\right|^{\prime}\right. \\
v=\frac{1}{4}, \frac{1}{2}, \frac{3}{4},
\end{array}
$$

Solving (6) for the independent solution, gives continuous hybrid method

$$
y_{n+j}=\sum_{i=0}^{3} \frac{(j h)^{(i)}}{i !} y_{n}^{(i)}+h^{4}\left[\sum_{k=0}^{1} \delta_{k} f_{n+k}+\delta_{v} f_{n+v}\right],
$$

where

$$
\delta_{0}=\frac{1}{7560}\left(48 t^{8}-240 t^{7}+490 t^{6}-525 t^{5}+315 t^{4}\right)
$$




$$
\begin{gathered}
\delta_{\frac{1}{4}}=-\frac{1}{945}\left(24 t^{8}-108 t^{7}+182 t^{6}-126 t^{5}\right) \\
\delta_{\frac{1}{2}}=\frac{1}{630}\left(24 t^{8}-96 t^{7}+133 t^{6}-63 t^{5}\right) \\
\delta_{\frac{3}{4}}=-\frac{1}{945}\left(24 t^{8}-84 t^{7}+98 t^{6}-42 t^{5}\right) \\
\delta_{1}=\frac{1}{7560}\left(48 t^{8}-144 t^{7}+154 t^{6}-63 t^{5}\right)
\end{gathered}
$$

Evaluating (7) at $t=\frac{1}{4}\left(\frac{1}{4}\right) 1$ gives a discrete block method

$$
A^{(0)} Y_{m}^{(i)}=\sum_{i}^{3} h^{(i)} e_{i} y_{n}^{(i)}+h^{(4-i)}\left[d_{i} f\left(y_{n}\right)+b_{i} f\left(Y_{m}\right)\right]
$$

where

$$
\begin{aligned}
Y_{m}^{(i)} & =\left[\begin{array}{llll}
y_{n+\frac{1}{4}}^{(i)} & y_{n+\frac{1}{2}}^{(i)} & y_{n+\frac{3}{4}}^{(i)} & y_{n+1}^{(i)}
\end{array}\right]^{T}, \\
f\left(Y_{m}\right) & =\left[\begin{array}{llll}
f_{n+\frac{1}{4}} & f_{n+\frac{1}{2}} & f_{n+\frac{3}{4}} & f_{n+1}
\end{array}\right]^{T}, \\
f\left(y_{n}\right) & =\left[\begin{array}{llll}
f_{n+\frac{1}{4}} & f_{n+\frac{1}{2}} & f_{n+\frac{3}{4}} & f_{n+1}
\end{array}\right]^{T},
\end{aligned}
$$

where $A^{(0)}=4 \times 4$ identity matrix.

When $i=0$ :

$$
\begin{gathered}
e_{0}=\left[\begin{array}{llll}
0 & 0 & 0 & 1 \\
0 & 0 & 0 & 1 \\
0 & 0 & 0 & 1 \\
0 & 0 & 0 & 1
\end{array}\right], e_{1}=\left[\begin{array}{llll}
0 & 0 & 0 & \frac{1}{4} \\
0 & 0 & 0 & \frac{1}{2} \\
0 & 0 & 0 & \frac{3}{4} \\
0 & 0 & 0 & 1
\end{array}\right], e_{2}=\left[\begin{array}{cccc}
0 & 0 & 0 & \frac{1}{32} \\
0 & 0 & 0 & \frac{1}{8} \\
0 & 0 & 0 & \frac{9}{32} \\
0 & 0 & 0 & \frac{1}{2}
\end{array}\right], \\
e_{3}=\left[\begin{array}{cccc}
0 & 0 & 0 & \frac{1}{384} \\
0 & 0 & 0 & \frac{1}{48} \\
0 & 0 & 0 & \frac{27}{384} \\
0 & 0 & 0 & \frac{1}{6}
\end{array}\right],
\end{gathered}
$$

then

$$
d_{0}=\left[\begin{array}{cccc}
0 & 0 & 0 & \frac{3373}{30965760} \\
0 & 0 & 0 & \frac{37}{30240} \\
0 & 0 & 0 & \frac{5319}{1146880} \\
0 & 0 & 0 & \frac{11}{945}
\end{array}\right], b_{0}=\left[\begin{array}{cccc}
\frac{139}{1548288} & \frac{-283}{5160960} & \frac{179}{7741440} & \frac{-131}{30965760} \\
\frac{59}{30240} & \frac{-1}{1152} & \frac{11}{30240} & \frac{-1}{15120} \\
\frac{2889}{286720} & \frac{-1539}{573440} & \frac{81}{57344} & \frac{-297}{1146880} \\
\frac{4}{135} & \frac{-1}{315} & \frac{4}{945} & \frac{1}{1512}
\end{array}\right] .
$$


When $i=1$ :

$$
\begin{gathered}
e_{1}=\left[\begin{array}{llll}
0 & 0 & 0 & 1 \\
0 & 0 & 0 & 1 \\
0 & 0 & 0 & 1 \\
0 & 0 & 0 & 1
\end{array}\right], e_{2}=\left[\begin{array}{cccc}
0 & 0 & 0 & \frac{1}{4} \\
0 & 0 & 0 & \frac{1}{2} \\
0 & 0 & 0 & \frac{3}{4} \\
0 & 0 & 0 & 1
\end{array}\right], e_{3}=\left[\begin{array}{cccc}
0 & 0 & 0 & \frac{1}{32} \\
0 & 0 & 0 & \frac{1}{8} \\
0 & 0 & 0 & \frac{9}{32} \\
0 & 0 & 0 & \frac{1}{2}
\end{array}\right] \\
d_{1}=\left[\begin{array}{llll}
0 & 0 & 0 & \frac{367}{23040} \\
0 & 0 & 0 & \frac{53}{1440} \\
0 & 0 & 0 & \frac{147}{2560} \\
0 & 0 & 0 & \frac{7}{90}
\end{array}\right], b_{1}=\left[\begin{array}{cccc}
\frac{3}{128} & \frac{-47}{3840} & \frac{29}{5760} & \frac{-7}{7680} \\
\frac{1}{10} & \frac{-1}{48} & \frac{1}{90} & \frac{-1}{480} \\
\frac{117}{640} & \frac{27}{1280} & \frac{3}{128} & \frac{-9}{2560} \\
\frac{4}{15} & \frac{1}{15} & \frac{4}{45} & 0
\end{array}\right] .
\end{gathered}
$$

When $i=2$ :

$$
e_{2}=\left[\begin{array}{llll}
0 & 0 & 0 & 1 \\
0 & 0 & 0 & 1 \\
0 & 0 & 0 & 1 \\
0 & 0 & 0 & 1
\end{array}\right], e_{3}=\left[\begin{array}{cccc}
0 & 0 & 0 & \frac{1}{4} \\
0 & 0 & 0 & \frac{1}{2} \\
0 & 0 & 0 & \frac{3}{4} \\
0 & 0 & 0 & 1
\end{array}\right]
$$

then

$$
d_{0}=\left[\begin{array}{cccc}
0 & 0 & 0 & \frac{367}{23040} \\
0 & 0 & 0 & \frac{53}{1440} \\
0 & 0 & 0 & \frac{147}{2560} \\
0 & 0 & 0 & \frac{7}{90}
\end{array}\right], b_{0}=\left[\begin{array}{cccc}
\frac{3}{128} & \frac{-47}{3840} & \frac{29}{5760} & \frac{-7}{7680} \\
\frac{1}{10} & \frac{-1}{48} & \frac{1}{90} & \frac{-1}{480} \\
\frac{117}{640} & \frac{27}{1280} & \frac{3}{128} & \frac{-9}{2560} \\
\frac{4}{15} & \frac{1}{15} & \frac{4}{45} & 0
\end{array}\right]
$$

When $i=3$ :

$$
e_{3}=\left[\begin{array}{llll}
0 & 0 & 0 & 1 \\
0 & 0 & 0 & 1 \\
0 & 0 & 0 & 1 \\
0 & 0 & 0 & 1
\end{array}\right]
$$

then

$$
d_{0}=\left[\begin{array}{cccc}
0 & 0 & 0 & \frac{251}{2880} \\
0 & 0 & 0 & \frac{29}{360} \\
0 & 0 & 0 & \frac{37}{320} \\
0 & 0 & 0 & \frac{7}{90}
\end{array}\right], b_{0}=\left[\begin{array}{cccc}
\frac{323}{1440} & \frac{-11}{120} & \frac{53}{1440} & \frac{-19}{2880} \\
\frac{31}{90} & \frac{1}{15} & \frac{1}{90} & \frac{-1}{360} \\
\frac{51}{160} & \frac{9}{40} & \frac{21}{160} & \frac{-3}{320} \\
\frac{16}{45} & \frac{2}{15} & \frac{16}{45} & \frac{7}{90}
\end{array}\right]
$$

\subsection{Implementation of the Method}

In order to implement the block method, we consider prediction equation

$$
Y_{m}^{(0)}=\sum_{i=0}^{3} \frac{(j h)^{(i)}}{i !} y_{n}^{(i)}+h^{4} \sum_{\lambda=0}^{3} \frac{\delta^{\lambda}}{\delta x^{\lambda}} f\left(x, y, y^{\prime}, y^{\prime \prime}, y^{\prime \prime \prime}\right)
$$


Substituting (??) into (8) give the block method which is implemented as a simultaneous integration on mesh $\pi_{N}: a=x_{0}<x_{1}<\ldots<x_{n}<x_{n+1}<\ldots<$ $x_{N}=b$ where $\pi_{N}$ is a partition of $[a, b]$ and $h=\frac{(b-a)}{N}$ is the step-size of the partition of $\pi_{N}$.

\section{Analysis of the Block Method}

\subsection{Order of the Block Method}

We define the linear operator $L\{y(x): h\}$ on (8) when $i=0$ such that

$$
L\{y(x): h\}=A^{(0)} Y_{m}-\sum_{i=0}^{3} h^{(i)} e_{i} y_{n}^{(i)}-h^{(4-i)}\left[d_{i} f\left(y_{n}\right)-b_{i} f\left(Y_{m}\right)\right]
$$

Expanding (10) in Taylor series and comparing the coefficients of $\mathrm{h}$ gives

$$
L\{y(x): h\}=c_{0} y(x)+\ldots+c_{p+2} h^{(p+2)} y^{(p+2)}(x)+\ldots
$$

Definition 1. The linear operator $L$ and associated block (11) are said to be of order $\mathrm{p}$ if $c_{0}=c_{1}=\ldots=c_{p+1}=0$ and $c_{p+2} \neq 0$ is called the error constant and implies that the truncation error is given by

$$
t_{n+k}=c_{p+2} h^{(p+2)} y^{(p+2)}(x)+O\left(h^{p+3}\right)
$$

For our method, the order of the block is seven and the error constants are:

$$
\left[\frac{1469}{475634073600}, \frac{11}{232243200}, \frac{1107}{5872025600}, \frac{1}{3073600}\right] .
$$

\subsection{Consistency}

A block method is said to be consistent if it has order $p \geq 1$; hence our method is consistent.

\subsection{Zero Stability}

A block method is said to be zero stable if as $h \rightarrow 0$, the roots $r_{j}=1(1) k$ of the characteristics polynomial $\rho(r)=0$ such that $\rho(r)=\left|\sum A^{(0)} R^{k-1}\right|$ satisfying $|R| \leq 1$ must have simplicity equal to unity. 
For our method

$$
\rho(r)=\left|R\left[\begin{array}{llll}
1 & 0 & 0 & 0 \\
0 & 1 & 0 & 0 \\
0 & 0 & 1 & 0 \\
0 & 0 & 0 & 1
\end{array}\right]-\left[\begin{array}{llll}
0 & 0 & 0 & 1 \\
0 & 0 & 0 & 1 \\
0 & 0 & 0 & 1 \\
0 & 0 & 0 & 1
\end{array}\right]\right|
$$

$r=0.0 .0 .1$

Hence our method is zero stable.

\subsection{Convergence}

A block method is said to be convergent if and only if it is consistent and zero stable. It has been clearly shown that our method is convergent.

\section{Numerical Experiment}

\subsection{Numerical Examples}

Error means $\left|y(x)-y\left(x_{n}\right)\right| ; y(x)$ is the exact result, $y\left(x_{n}\right)$ is the computed result.

ERJ is absolute error in [5].

ERA is Error in [14].

Problem 1. We consider the linear initial value problem

$$
\begin{gathered}
y^{i v}=y^{\prime \prime \prime}+y^{\prime \prime}+y^{\prime}+2 y \\
y(0)=0, y^{\prime}(0)=0, y^{\prime \prime}(0)=0, y^{\prime \prime \prime}(0)=30,0 \leq x \leq 2
\end{gathered}
$$

Exact solution $y(x)=2 e^{2 x}-5 e^{-x}+3 \cos x-9 \sin x$

Source: [5]

The theoretical solution at $x=2$ is $y(2) \simeq 99.0975062990331510$.

The errors in the solution were obtained at $x=2$ using our new block method for a fixed step-size $h=\frac{1}{10}$

We compare our results with the results of [5]where four step method of order six is developed.

Problem II. We consider a non linear problem

$$
y^{i v}=\left(y^{\prime}\right)^{2}-y y^{\prime \prime \prime}-4 x^{2}+e^{x}\left(1-4 x+x^{2}\right), 0 \leq x \leq 1
$$




\begin{tabular}{cccc}
\hline Steps & $y$ & Error & ERJ \\
\hline 20 & 99.08746838807877 & $1.946023(-05)$ & $1.25612(-04)$ \\
40 & 99.08750563415182 & $6.648812(-07)$ & $1.90752(-06)$ \\
60 & 99.08750620883327 & $9.020016(-08)$ & $1.66517(-07)$ \\
80 & 99.08750627730742 & $2.172474(-08)$ & $2.96411(-08)$ \\
100 & 99.08750629185001 & $7.183132(-09)$ & $8.65117(-08)$ \\
\hline
\end{tabular}

Table 1: Results of Problem 1

\begin{tabular}{ccccc}
\hline$x$ & Exact results & Computed result & Error & ERA \\
\hline 0.1 & 1.1151709180756 & 1.1151709180756 & $0.000000(+00)$ & $9.0460(-13)$ \\
0.2 & 1.2614027581601 & 1.2614027581601 & $4.440892(-16)$ & $2.1516(-12)$ \\
0.3 & 1.4398588075760 & 1.4398588075760 & $4.440892(-16)$ & $3.7549(-12)$ \\
0.4 & 1.6518246976412 & 1.6518246976412 & $4.440892(-16)$ & $5.6885(-12)$ \\
0.5 & 1.8987212707001 & 1.8987212707001 & $6.661338(-16)$ & $7.8819(-12)$ \\
0.6 & 2.1821188003905 & 2.1821188003905 & $4.440892(-16)$ & $1.0212(-11)$ \\
0.7 & 2.5037527074704 & 2.5037527074704 & $4.440892(-16)$ & $1.2497(-11)$ \\
0.8 & 2.8655409284924 & 2.8655409284924 & $8.881784(-16)$ & $1.4486(-11)$ \\
0.9 & 3.2696031111569 & 3.2696031111569 & $3.108624(-15)$ & $1.5849(-11)$ \\
1.0 & 3.7182818284590 & 3.7182818284590 & $6.217249(-15)$ & $1.6159(-11)$ \\
\hline
\end{tabular}

Table 2: Results of Problem II

$$
, y(0)=1, y^{\prime}(0)=y^{\prime \prime}(0)=3, y^{\prime \prime}(0)=1 .
$$

Exact solution $y(x)=x^{2}+e^{x}$

Source: [14]

[14]solved this problem using 5-step block method of order eight.

Problem III. We consider a linear problem

$$
\begin{gathered}
y^{i v}+y^{\prime \prime}=0,0 \leq x \leq \frac{\pi}{2} \\
y(0)=0, y^{\prime}(0)=\frac{1.1}{72-50 \pi}, y^{\prime \prime}(0)=\frac{-1.1}{144-100 \pi}, y^{\prime \prime}(0)=\frac{1.2}{144-100 \pi}
\end{gathered}
$$

Exact solution $y(x)=\frac{1-x-\cos x-1.2 \sin x}{144-100 \pi}$

Source: [14]

[14] solved this problem using 5 -step block method of order eight. 


\begin{tabular}{ccccc}
\hline$X$ & Exact result & Computed result & Error & ERA \\
\hline 0.1 & $1.2623718420(-03)$ & $1.2623718420(-03)$ & $4.336809(-19)$ & $6.5052(-19)$ \\
0.2 & $2.4592829189(-03)$ & $2.4592829189(-03)$ & $0.000000(+00)$ & $1.3010(-18)$ \\
0.3 & $3.5846460422(-03)$ & $3.5846460422(-03)$ & $0.000000(+00)$ & $4.7704(-18)$ \\
0.4 & $4.6330889070(-03)$ & $4.6330889070(-03)$ & $2.602085(-18)$ & $1.7347(-17)$ \\
0.5 & $5.6000077703(-03)$ & $5.6000077703(-03)$ & $6.938894(-18)$ & $4.3368(-17)$ \\
0.6 & $6.4816134499(-03)$ & $6.4816134499(-03)$ & $4.336809(-18)$ & $9.5409(-17)$ \\
0.7 & $7.2749691846(-03)$ & $7.2749691846(-03)$ & $6.071532(-18)$ & $1.8127(-16)$ \\
0.8 & $7.9780199777(-03)$ & $7.9780199777(-03)$ & $1.040834(-17)$ & $3.1571(-16)$ \\
0.9 & $8.5896131294(-03)$ & $8.5896131294(-03)$ & $8.673617(-17)$ & $5.1868(-16)$ \\
1.0 & $9.1095097546(-03)$ & $9.1095097546(-03)$ & $1.214356(-17)$ & $8.0491(-16)$ \\
\hline
\end{tabular}

Table 3: Results of Problem III

\section{Discussion of Result}

We considered three numerical examples, the results re-affirms the claim of [3, 13] that hybrid methods give better results than the k-step methods especially when the method is of low step-length. It equally shows that block method gives better approximation than the predictor-corrector method, though we did not show the comparison our results with any predictor-corrector method but [14] comfirmed the supremacy of their results over predictor-corrector.

\section{Conclusion}

We have presented the development of method that is cost effective in terms of cost of implementation, time of development and execution, and approximation. The method developed in this paper adopted Taylor's series expansion of order three as the starting value; hence this method is non-self starting block method [15].

\section{References}

[1] D. O. Awoyemi, E. A. Adebile, A. O. Adesanya, T. A. Anake. Modified block method for the direct solution of second order ordinary differential equations. IJPAM. 3(3), 2011, 181-188. 
[2] D. O. Awoyemi. Algorithmic approach for the direct solution of second order initial value problems of ordinary differential equations. INT J COMPUT MATH. J. of Comp. Math., 82(3), 2005, 321-329.

[3] A. O. Adesanya, M. O. Udoh, A. M. Ajileye. A new hybrid block method for the solution of general third order initial value problems of ordinary differential equations. IJPAM. 86(2), 2013, 37-48. doi:http://dx.doi.org/10.12732/ijpam.v86i2.11.

[4] S. J. Kayode, F. O. Obaruah. Continuous y-function hybrid method for the direct solution of differential equations. IJDEA, 12(1), 2013, 365-375.

[5] S. N. Jator. On a class of hybrid method for $y^{\prime \prime}=f\left(x, y, y^{\prime}\right)$. IJPAM. 59(4), 2010, 381-395.

[6] A. O. Adesanya, M. R. Odekunle, M. O. Udoh. Four step continuous method for the solution of $y^{\prime \prime}=f\left(x, y, y^{\prime}\right)$, AJCM. 3, 2013, 169-174. doi:10.4236/ajcm.2013.32025.

[7] A. B. Sofoluwe, S. A. Okunuga, J. O. Ehigie. Composite multiderivative multistep backward differentiation formula for solving stiff initial value problems, J. of Maths. Sci. 2(1), 2013, 227-239.

[8] N. Waeleh, Z. A. Majid, F. Ismail. A new algorithms for solving higher order IVP of ODEs, Applied Mathematical Sciences, 5(56), 2011, 27952805.

[9] S. J. Kayode, O. Adeyeye. A 3-step hybrid method for the direct solution of second order initial value problems, AJBAS. 5(12), 2011, 2121-2126.

[10] S. N. Jator. A sixth order linear multistep method for direct solution of $y^{\prime \prime}=f\left(x, y, y^{\prime}\right)$. IJPAM. 4(1), 2007, 457-472.

[11] A. A. James, A. O. Adesanya. M. K. Fasansi, Starting order seven method accurately for the solution of first order initial value problems of ordinary differential equations. JPAM. 6(1), 2013, 30-39. doi:10.3968/j.pam.1925252820130601.5231.

[12] A. A. James, A. O. Adesanya, S. Joshua. Continuous block method for the solution of second order initial value problems of ordinary differential equations. IJPAM. 83(3), 2013, 405-416. doi:http://doi.org/10.12732/ijpam.v8313.3. 
[13] T. A. Anake, D. O. Awoyemi, A. O. Adesanya. One step implicit hybrid block method for the direct solution of general second order ordinary differential equations. IJAM. 42(4), 2012.

[14] A. O. Adesanya, A. A. Momoh, A. M. Alkali, A. Tahir. Five steps block method for the solution of fourth order ordinary differential equations, IJERA. 2(5), 2012, 991-998.

[15] S. H. Abbass, Derivation of a new block method similar to the block trapezoidal rule for the numerical solution of first order IVPs, Science Echoes, 2, 2006,10-24. 
